\author{
MAMORU NUNOKAWA, EMEL YAVUZ DUMAN \\ and SHIGEYOSHI OWA
}

\title{
Properties of functions concerned with Carathéodory functions
}

\begin{abstract}
Let $\mathcal{P}_{n}$ denote the class of analytic functions $p(z)$ of the form $p(z)=1+c_{n} z^{n}+c_{n+1} z^{n+1}+\ldots$ in the open unit disc $\mathbb{U}$. Applying the result by S. S. Miller and P. T. Mocanu (J. Math. Anal. Appl. 65 (1978), 289-305), some interesting properties for $p(z)$ concerned with Carathéodory functions are discussed. Further, some corollaries of the results concerned with the result due to M. Obradović and S. Owa (Math. Nachr. 140 (1989), 97-102) are shown.
\end{abstract}

1. Introduction. Let $\mathcal{A}_{n}$ denote the class of functions $f(z)$ of the form

$$
f(z)=z+\sum_{k=n+1}^{\infty} a_{k} z^{k} \quad(n=1,2,3, \ldots)
$$

which are analytic in the open unit disc $\mathbb{U}=\{z \in \mathbb{C}|| z \mid<1\}$. If a function $f(z) \in \mathcal{A}_{n}$ satisfies

$$
\operatorname{Re}\left(\frac{z f^{\prime}(z)}{f(z)}\right)>0 \quad(z \in \mathbb{U}),
$$

then $f(z)$ is said to be starlike with respect to the origin in $\mathbb{U}$. We denote by $\mathcal{S}_{n}^{*}$ the subclass of $\mathcal{A}_{n}$ consisting of functions $f(z)$ which are starlike with respect to the origin in $\mathbb{U}$. From the definition of the class $\mathcal{S}_{n}^{*}$, we see that

2000 Mathematics Subject Classification. Primary 30C45.

Key words and phrases. Analytic, starlike, Carathéodory function. 
if $f(z) \in \mathcal{A}_{n}$ satisfies

$$
\left|\frac{z f^{\prime}(z)}{f(z)}-1\right|<1 \quad(z \in \mathbb{U}),
$$

then $f(z) \in \mathcal{S}_{n}^{*}$. We denote by $\mathcal{T}_{n}^{*}$ the subclass of $\mathcal{S}_{n}^{*}$ consisting of $f(z)$ satisfying (1.3).

Obradović and Owa [5] have shown the following result:

Theorem A. If $f(z) \in \mathcal{A}_{1}$ satisfies $f(z) f^{\prime}(z) \neq 0$ for $0<|z|<1$ and

$$
\left|1+\frac{z f^{\prime \prime}(z)}{f^{\prime}(z)}\right|<\frac{5}{4}\left|\frac{z f^{\prime}(z)}{f(z)}\right| \quad(z \in \mathbb{U}),
$$

then $f(z) \in \mathcal{T}_{1}^{*}$.

In order to discuss our results, we have to recall here the following lemma due to Miller and Mocanu [3] (also due to Jack [2]):

Lemma 1.1. Let

$$
w(z)=a_{n} z^{n}+a_{n+1} z^{n+1}+\ldots \quad\left(a_{n} \neq 0\right)
$$

be analytic in $\mathbb{U}$. If there exists a point $z_{0} \in \mathbb{U}$ on the circle $|z|=r<1$ such that

$$
\max _{|z| \leq\left|z_{0}\right|}|w(z)|=\left|w\left(z_{0}\right)\right|
$$

then we can write

$$
z_{0} w^{\prime}\left(z_{0}\right)=m w\left(z_{0}\right)
$$

where $m$ is real and $m \geq n$.

Example 1.1. We consider the function $w(z)$ given by

$$
w(z)=z^{n}+\frac{e^{i \theta}}{n+1} z^{n+1} \quad(n=1,2,3, \ldots) .
$$

Then, it follows that

$$
\max _{|z| \leq\left|z_{0}\right|}|w(z)|=\max _{|z| \leq\left|z_{0}\right|}|z|^{n}\left|1+\frac{e^{i \theta} z}{n+1}\right| \leq r^{n}\left(1+\frac{r}{n+1}\right)
$$

for $z_{0}=r e^{-i \theta} \in \mathbb{U}$. This shows that $|w(z)|$ attains its maximum value at a point $z_{0} \in \mathbb{U}$ on the circle $|z|=r$. For such a point $z_{0}=r e^{-i \theta}$, we have that

$$
\frac{z_{0} w^{\prime}\left(z_{0}\right)}{w\left(z_{0}\right)}=\frac{z_{0}^{n}\left(n+e^{i \theta} z_{0}\right)}{z_{0}^{n}\left(1+\frac{e^{i \theta} z_{0}}{n+1}\right)}=\frac{(n+1)(n+r)}{n+1+r}=m \geq n .
$$


Let $\mathcal{P}_{n}$ be the class of functions $p(z)$ of the form

$$
p(z)=1+\sum_{k=n}^{\infty} c_{k} z^{k} \quad\left(c_{n} \neq 0\right)
$$

which are analytic in $\mathbb{U}$. We also denote by $\mathcal{Q}_{n}$ the subclass of $\mathcal{P}_{n}$ consisting of $f(z)$ which satisfy

$$
|p(z)-1|<1 \quad(z \in \mathbb{U}) .
$$

Since $p(z) \in \mathcal{Q}_{n}$ shows that $\operatorname{Re} p(z)>0(z \in \mathbb{U}), p(z) \in \mathcal{Q}_{n}$ is said to be a Carathéodory function in $\mathbb{U}$ (see Carathéodory [1]).

2. Conditions for the classes $\mathcal{Q}_{n}$ and $\mathcal{T}_{n}^{*}$. Applying Lemma 1.1, we discuss some conditions for $p(z) \in \mathcal{P}_{n}$ to be in the class $\mathcal{Q}_{n}$.

Theorem 2.1. If $p(z) \in \mathcal{P}_{n}$ satisfies

$$
\operatorname{Re}\left(p(z)+\alpha \frac{z p^{\prime}(z)}{p(z)}\right)<\sqrt{\alpha n}|p(z)| \quad(z \in \mathbb{U})
$$

for some real $\alpha>0$, then $p(z) \in \mathcal{Q}_{n}$.

Proof. Note that $p(z) \neq 0(z \in \mathbb{U})$ with the condition (2.1). Let us define the function $w(z)$ by

$$
p(z)=1+w(z) \quad(z \in \mathbb{U})
$$

for $p(z) \in \mathcal{P}_{n}$. Then $w(z)$ is analytic in $\mathbb{U}$ and

$$
w(z)=c_{n} z^{n}+c_{n+1} z^{n+1}+\ldots .
$$

It follows that

$$
p(z)+\alpha \frac{z p^{\prime}(z)}{p(z)}=1+w(z)+\frac{\alpha z w^{\prime}(z)}{1+w(z)}
$$

and that

$$
\begin{aligned}
\frac{1}{|p(z)|} \operatorname{Re} & \left(p(z)+\alpha \frac{z p^{\prime}(z)}{p(z)}\right) \\
= & \frac{1}{|1+w(z)|} \operatorname{Re}\left(1+w(z)+\frac{\alpha z w^{\prime}(z)}{1+w(z)}\right)<\sqrt{\alpha n}
\end{aligned}
$$

for $z \in \mathbb{U}$.

We suppose that there exists a point $z_{0} \in \mathbb{U}$ such that

$$
\max _{|z| \leq\left|z_{0}\right|}|w(z)|=\left|w\left(z_{0}\right)\right|=1 .
$$


Then, Lemma 1.1 gives us that $w\left(z_{0}\right)=e^{i \theta}$ and $z_{0} w^{\prime}\left(z_{0}\right)=m e^{i \theta}(m \geq n)$. For such a point $z_{0}$, we have that

$$
\begin{aligned}
\frac{1}{\left|p\left(z_{0}\right)\right|} \operatorname{Re}\left(p\left(z_{0}\right)+\alpha \frac{z_{0} p^{\prime}\left(z_{0}\right)}{p\left(z_{0}\right)}\right) & =\frac{1}{\mid 1+e^{i \theta \mid}} \operatorname{Re}\left(1+e^{i \theta}+\frac{\alpha m e^{i \theta}}{1+e^{i \theta}}\right) \\
& =\frac{1}{\sqrt{2(1+\cos \theta)}}\left(1+\cos \theta+\frac{\alpha m}{2}\right) \\
& =\frac{1}{\sqrt{2}}\left(\sqrt{1+\cos \theta}+\frac{\alpha m}{2 \sqrt{1+\cos \theta}}\right) \\
& \geq \sqrt{\alpha m} \geq \sqrt{\alpha n} .
\end{aligned}
$$

This contradicts the condition (2.1). Therefore, there is no such point $z_{0} \in$ $\mathbb{U}$. This means that $p(z) \in \mathcal{Q}_{n}$.

Corollary 2.1. If $f(z) \in \mathcal{A}_{n}$ satisfies $f(z) f^{\prime}(z) \neq 0$ for $0<|z|<1$ and

$$
\operatorname{Re}\left\{(1-\alpha) \frac{z f^{\prime}(z)}{f(z)}+\alpha\left(1+\frac{z f^{\prime \prime}(z)}{f^{\prime}(z)}\right)\right\}<\sqrt{\alpha n}\left|\frac{z f^{\prime}(z)}{f(z)}\right| \quad(z \in \mathbb{U})
$$

for some real $\alpha>0$, then $f(z) \in \mathcal{T}_{n}^{*}$.

Proof. Letting $p(z)=\frac{z f^{\prime}(z)}{f(z)}$ in Theorem 2.1, we have that

$$
p(z)+\alpha \frac{z p^{\prime}(z)}{p(z)}=(1-\alpha) \frac{z f^{\prime}(z)}{f(z)}+\alpha\left(1+\frac{z f^{\prime \prime}(z)}{f^{\prime}(z)}\right) .
$$

The proof of the corollary follows from the above.

Next we derive

Theorem 2.2. If $p(z) \in \mathcal{P}_{n}$ satisfies $\operatorname{Re} p(z) \neq 0(z \in \mathbb{U})$ and

$$
\operatorname{Re}\left(p(z)+\alpha \frac{z p^{\prime}(z)}{p(z)}\right)<\left(1+\frac{\alpha n}{4}\right) \operatorname{Re} p(z) \quad(z \in \mathbb{U})
$$

for some real $\alpha>0$, then $p(z) \in \mathcal{Q}_{n}$.

Proof. Define the function $w(z)$ by $(2.2)$ for $p(z) \in \mathcal{P}_{n}$. Then, $w(z)$ is analytic in $\mathbb{U}$,

$$
w(z)=c_{n} z^{n}+c_{n+1} z^{n+1}+\ldots,
$$

and

$$
\frac{\operatorname{Re}\left(p(z)+\alpha \frac{z p^{\prime}(z)}{p(z)}\right)}{\operatorname{Re} p(z)}=\frac{\operatorname{Re}\left(1+w(z)+\frac{\alpha z w^{\prime}(z)}{1+w(z)}\right)}{\operatorname{Re}(1+w(z))}<1+\frac{\alpha n}{4}
$$

$(z \in \mathbb{U})$. If we suppose that there exists a point $z_{0} \in \mathbb{U}$ on the circle $|z|=r<1$ such that

$$
\max _{|z| \leq\left|z_{0}\right|}|w(z)|=\left|w\left(z_{0}\right)\right|=1
$$


we can write that $w\left(z_{0}\right)=e^{i \theta}$ and $z_{0} w^{\prime}\left(z_{0}\right)=m e^{i \theta}$. This shows that

$$
\frac{\operatorname{Re}\left(p\left(z_{0}\right)+\alpha \frac{z_{0} p^{\prime}\left(z_{0}\right)}{p\left(z_{0}\right)}\right)}{\operatorname{Re} p\left(z_{0}\right)}=\frac{1+\cos \theta+\frac{\alpha m}{2}}{1+\cos \theta} \geq 1+\frac{\alpha m}{4} \geq 1+\frac{\alpha n}{4} .
$$

Since (2.11) contradicts our condition (2.9), $|w(z)|<1$ for all $z \in \mathbb{U}$. This means that $p(z) \in \mathcal{Q}_{n}$.

If we take $p(z)=\frac{z f^{\prime}(z)}{f(z)}$ in Theorem 2.2, we have

Corollary 2.2. If $f(z) \in \mathcal{A}_{n}$ satisfies $\operatorname{Re}\left(\frac{z f^{\prime}(z)}{f(z)}\right) \neq 0(z \in \mathbb{U})$ and

$$
\operatorname{Re}\left\{(1-\alpha) \frac{z f^{\prime}(z)}{f(z)}+\alpha\left(1+\frac{z f^{\prime \prime}(z)}{f^{\prime}(z)}\right)\right\}<\left(1+\frac{\alpha n}{4}\right) \operatorname{Re}\left(\frac{z f^{\prime}(z)}{f(z)}\right)
$$

$(z \in \mathbb{U})$ for some real $\alpha>0$, then $f(z) \in \mathcal{T}_{n}^{*}$.

Corollary 2.3. If $f(z) \in \mathcal{A}_{n}$ satisfies

$$
\operatorname{Re}\left(\frac{z f^{\prime \prime}(z)}{f^{\prime}(z)}\right)<\operatorname{Re}\left(\frac{z f^{\prime}(z)}{f(z)}\right)+\frac{n-2}{n} \quad(z \in \mathbb{U}),
$$

then $f(z) \in \mathcal{T}_{n}^{*}$.

Proof. If we write

$$
\frac{z f^{\prime}(z)}{f(z)}=1+w(z) \quad\left(f(z) \in \mathcal{A}_{n}\right)
$$

we see that $w(z)$ is analytic in $\mathbb{U}$ and

$$
w(z)=c_{n} z^{n}+c_{n+1} z^{n+1}+\ldots
$$

For such a function $w(z)$, we see that

$$
\operatorname{Re}\left(\frac{z f^{\prime \prime}(z)}{f^{\prime}(z)}-\frac{z f^{\prime}(z)}{f(z)}\right)=\operatorname{Re}\left(\frac{z w^{\prime}(z)}{1+w(z)}-1\right)<\frac{n-2}{2} \quad(z \in \mathbb{U})
$$

Supposing that there exists a point $z_{0} \in \mathbb{U}$ on the circle $|z|=r<1$ such that

$$
\max _{|z| \leq\left|z_{0}\right|}|w(z)|=\left|w\left(z_{0}\right)\right|=1,
$$

we can write that $w\left(z_{0}\right)=e^{i \theta}$ and $z_{0} w^{\prime}\left(z_{0}\right)=m e^{i \theta}$. Therefore, we have

$$
\operatorname{Re}\left(\frac{z_{0} f^{\prime \prime}\left(z_{0}\right)}{f^{\prime}\left(z_{0}\right)}-\frac{z_{0} f^{\prime}\left(z_{0}\right)}{f\left(z_{0}\right)}\right)=\operatorname{Re}\left(\frac{k e^{i \theta}}{1+e^{i \theta}}-1\right)=\frac{k}{2}-1 \geq \frac{n-2}{2}
$$

which contradicts the condition (2.13). This implies that $f(z) \in \mathcal{T}_{n}^{*}$. 
Example 2.1. Let us consider the function $p(z)$ given by

$$
p(z)=1+a_{n} z^{n} \quad(z \in \mathbb{U})
$$

for some $n \in \mathbb{N}=\{1,2,3, \ldots\}$, where $a_{n}$ satisfies

$$
a_{n}^{3}+2 a_{n}-1 \leq 0 \quad\left(0<a_{n}<1\right) .
$$

Then $p(z) \in \mathcal{P}_{n}$ and $p(z) \neq 0(z \in \mathbb{U})$. It is clear that $p(z)$ satisfies the condition (2.9) in Theorem 2.2 for $z=0$.

Let us put $z=e^{i \theta}$ for $p(z)$. Then we see that

$$
\operatorname{Re}\left(p(z)+\alpha \frac{z p^{\prime}(z)}{p(z)}\right)=1+a_{n} \cos n \theta+\frac{\alpha n a_{n}\left(a_{n}+\cos n \theta\right)}{a_{n}^{2}+1+2 a_{n} \cos n \theta}
$$

and

$$
\left(1+\frac{\alpha n}{4}\right) \operatorname{Re} p(z)=\left(1+\frac{\alpha n}{4}\right)\left(1+a_{n} \cos n \theta\right) .
$$

This gives us that

$$
\begin{aligned}
\left(1+\frac{\alpha n}{4}\right) \operatorname{Re} p(z) & -\operatorname{Re}\left(p(z)+\alpha \frac{z p^{\prime}(z)}{p(z)}\right) \\
& =\frac{\alpha n\left(1+2 a_{n} \cos n \theta+a_{n}^{3} \cos n \theta+2 a_{n}^{2} \cos ^{2} n \theta\right)}{4\left(a_{n}^{2}+1+2 a_{n} \cos n \theta\right)} \\
& \geq \frac{\alpha n\left(1-2 a_{n}-a_{n}^{3}\right)}{4\left(a_{n}^{2}+1+2 a_{n} \cos n \theta\right)} \geq 0 .
\end{aligned}
$$

Therefore, the function $p(z)$ satisfies the condition (2.9) for all $z \in \mathbb{U}$. Indeed, we see that

$$
|p(z)-1|=\left|a_{n} z^{n}\right|<a_{n}<1 \quad(z \in \mathbb{U}) .
$$

Furthermore, if we define the function $f(z) \in \mathcal{A}_{n}$ by

$$
\frac{z f^{\prime}(z)}{f(z)}=1+a_{n} z^{n}
$$

with some real $a_{n}\left(0<a_{n}<1\right)$ satisfying

$$
a_{n}^{3}+2 a_{n}-1 \leq 0,
$$

then we have that

$$
f(z)=z e^{\frac{a_{n}}{n} z^{n}}
$$

which satisfies the condition (2.12) in Corollary 2.2.

If we consider the function

$$
g(x)=x^{3}+2 x-1 \quad(0<x<1),
$$

we see that $g(0)=-1<0$ and $g\left(\frac{1}{2}\right)=\frac{1}{8}>0$. Therefore, there exists some real $x(0<x<1)$ such that $g(x) \leq 0$. Indeed, we see that

$$
0.4533<x<0.4534 \text {. }
$$


3. Properties for the classes $\mathcal{P}_{n}$ and $\mathcal{A}_{n}$. We discuss some properties for functions in the classes $\mathcal{P}_{n}$ and $\mathcal{A}_{n}$.

Theorem 3.1. If $p(z) \in \mathcal{P}_{n}$ satisfies

$$
\int_{|z|=r}\left|\operatorname{Re}\left(\frac{z p^{\prime}(z)}{p(z)}\right)\right| d \theta<\pi
$$

for $z=r e^{i \theta}(0<r<1)$, then $\operatorname{Re} p(z)>0(z \in \mathbb{U})$.

Proof. It follows from (3.1) that

$$
\int_{|z|=r}\left|\operatorname{Re}\left(\frac{z p^{\prime}(z)}{p(z)}\right)\right| d \theta=\int_{0}^{2 \pi}\left|\frac{d \arg p(z)}{d \theta}\right| d \theta=\int_{|z|=r}|d \arg p(z)|<\pi .
$$

This implies that $\operatorname{Re} p(z)>0$ for $|z|=r<1$. Applying the maximum principle for harmonic functions, we obtain that $\operatorname{Re} p(z)>0(z \in \mathbb{U})$.

From Theorem 3.1, we have

Corollary 3.1. If $f(z) \in \mathcal{A}_{n}$ satisfies

$$
\int_{|z|=r}\left|\operatorname{Re}\left(1+\frac{z f^{\prime \prime}(z)}{f^{\prime}(z)}-\frac{z f^{\prime}(z)}{f(z)}\right)\right| d \theta<\pi
$$

for $z=r e^{i \theta}(0<r<1)$, then $f(z) \in \mathcal{S}_{n}^{*}$.

Further, applying the same method as the proof by Umezawa [5] and Nunokawa [3], we derive the following result:

Theorem 3.2. If $f(z) \in \mathcal{A}_{1}$ satisfies

$$
-\frac{\beta}{4 \beta-1}<\operatorname{Re}\left(\frac{z f^{\prime \prime}(z)}{f^{\prime}(z)}\right)<\beta \quad(z \in \mathbb{U})
$$

for some real $\beta \geq \frac{1}{4}$, then $\operatorname{Re} f^{\prime}(z)>0(z \in \mathbb{U})$.

Proof. We note that if $f^{\prime}\left(z_{0}\right)=0$ for some $z_{0} \in \mathbb{U}$, then $f(z)$ does not satisfy the condition (3.4). This shows that $f^{\prime}(z) \neq 0$ for all $z \in \mathbb{U}$. Applying the same method by Umezawa [5] and Nunokawa [3], we have that

$$
\int_{|z|=r} \frac{z f^{\prime \prime}(z)}{f^{\prime}(z)} d \theta=\int_{|z|=r} \frac{z f^{\prime \prime}(z)}{f^{\prime}(z)} \frac{d z}{i z}=-i \int_{|z|=r} \frac{z f^{\prime \prime}(z)}{f^{\prime}(z)} d z=0 .
$$

We denote by $\mathcal{C}_{1}$ the part of the circle $|z|=r$ on which

$$
\operatorname{Re}\left(\frac{z f^{\prime \prime}(z)}{f^{\prime}(z)}\right) \geq 0
$$

and

$$
\int_{\mathcal{C}_{1}} d \arg z=x .
$$


On the other hand, let us denote by $\mathcal{C}_{2}$ the part of the circle $|z|=r$ on which

$$
\operatorname{Re}\left(\frac{z f^{\prime \prime}(z)}{f^{\prime}(z)}\right)<0
$$

and

$$
\int_{\mathcal{C}_{2}} d \arg z=2 \pi-x
$$

Putting

$$
y_{1}=\int_{\mathcal{C}_{1}} \operatorname{Re}\left(\frac{z f^{\prime \prime}(z)}{f^{\prime}(z)}\right) d \theta=\int_{\mathcal{C}_{1}}\left(\frac{d \arg f^{\prime}(z)}{d \theta}\right) d \theta
$$

and

$$
-y_{2}=\int_{\mathcal{C}_{2}} \operatorname{Re}\left(\frac{z f^{\prime \prime}(z)}{f^{\prime}(z)}\right) d \theta=\int_{\mathcal{C}_{2}}\left(\frac{d \arg f^{\prime}(z)}{d \theta}\right) d \theta,
$$

we have that $y_{1}-y_{2}=0$.

In view of the condition (3.4), we obtain that

$$
y_{1}<\beta x \text { and } y_{2}<\frac{\beta}{4 \beta-1}(2 \pi-x) .
$$

If $y_{1} \geq \frac{\pi}{2}$, then $y_{2}=y_{1} \geq \frac{\pi}{2}$ and $\frac{\pi}{2}<\beta x$. On the other hand, we have that

$$
y_{2}<\frac{\beta}{4 \beta-1}(2 \pi-x)<\frac{2 \pi \beta-\frac{\pi}{2}}{4 \beta-1}=\frac{\pi}{2} .
$$

This contradicts the inequality $y_{2} \geq \frac{\pi}{2}$. Therefore, $y_{1}=y_{2}<\frac{\pi}{2}$. Consequently, we obtain that

$$
y_{1}+y_{2}=\int_{|z|=r}\left|\operatorname{Re}\left(\frac{z f^{\prime \prime}(z)}{f^{\prime}(z)}\right)\right| d \theta=\int_{|z|=r}\left|d \arg f^{\prime}(z)\right|<\pi,
$$

which implies that $\operatorname{Re} f^{\prime}(z)>0(z \in \mathbb{U})$.

Finally, letting $\beta \rightarrow \infty, \beta=\frac{1}{4}$ and $\beta=\frac{1}{2}$ in Theorem 3.2, we have the following corollary.

Corollary 3.2. If $f(z) \in \mathcal{A}_{1}$ satisfies one of the following conditions

$$
\begin{aligned}
& \operatorname{Re}\left(\frac{z f^{\prime \prime}(z)}{f^{\prime}(z)}\right)>-\frac{1}{4} \quad(z \in \mathbb{U}), \\
& \operatorname{Re}\left(\frac{z f^{\prime \prime}(z)}{f^{\prime}(z)}\right)<\frac{1}{4} \quad(z \in \mathbb{U}), \\
& \left|\operatorname{Re}\left(\frac{z f^{\prime \prime}(z)}{f^{\prime}(z)}\right)\right|<1 \quad(z \in \mathbb{U}),
\end{aligned}
$$

then $\operatorname{Re} f^{\prime}(z)>0(z \in \mathbb{U})$. 


\section{REFERENCES}

[1] Carathéodory, C., Über den Variabilitatsbereich der Koeffizienten von Potenzreihen, die gegebene Werte nicht annehmen, Math. Ann. 64(1907), 95-115.

[2] Jack, I. S., Functions starlike and convex of order $\alpha$, J. London Math. Soc. 3 (1971), 469-474.

[3] Miller, S. S., Mocanu, P. T., Second order differential inequalities in the complex plane, J. Math. Anal. Appl. 65 (1978), 289-305.

[4] Nunokawa, M., On the Bazilević analytic functions, Sci. Rep. Fac. Edu. Gunma Univ. 21 (1972), 9-13.

[5] Obradović, M., Owa, S., A criterion for starlikeness, Math. Nachr. 140 (1989), 97102.

[6] Umezawa, T., Analytic functions convex in one direction, J. Math. Soc. Japan 4 (1952), 194-202.

Mamoru Nunokawa

Emeritus Professor of University of Gunma

Hoshikuki 798-8, Chuou-Ward

Chiba, Chiba 260-0808

Japan

e-mail: mamoru_nuno@doctor.nifty.jp

Emel Yavuz Duman

Department of Mathematics and Computer Science

İstanbul Kültür University

34156 Bakıröy, İstanbul

Turkey

e-mail: e.yavuz@iku.edu.tr

Shigeyoshi Owa

Department of Mathematics

Kinki University

Higashi-Osaka, Osaka 577-8502

Japan

e-mail: shige21@ican.zaq.ne.jp

Received April 11, 2012 